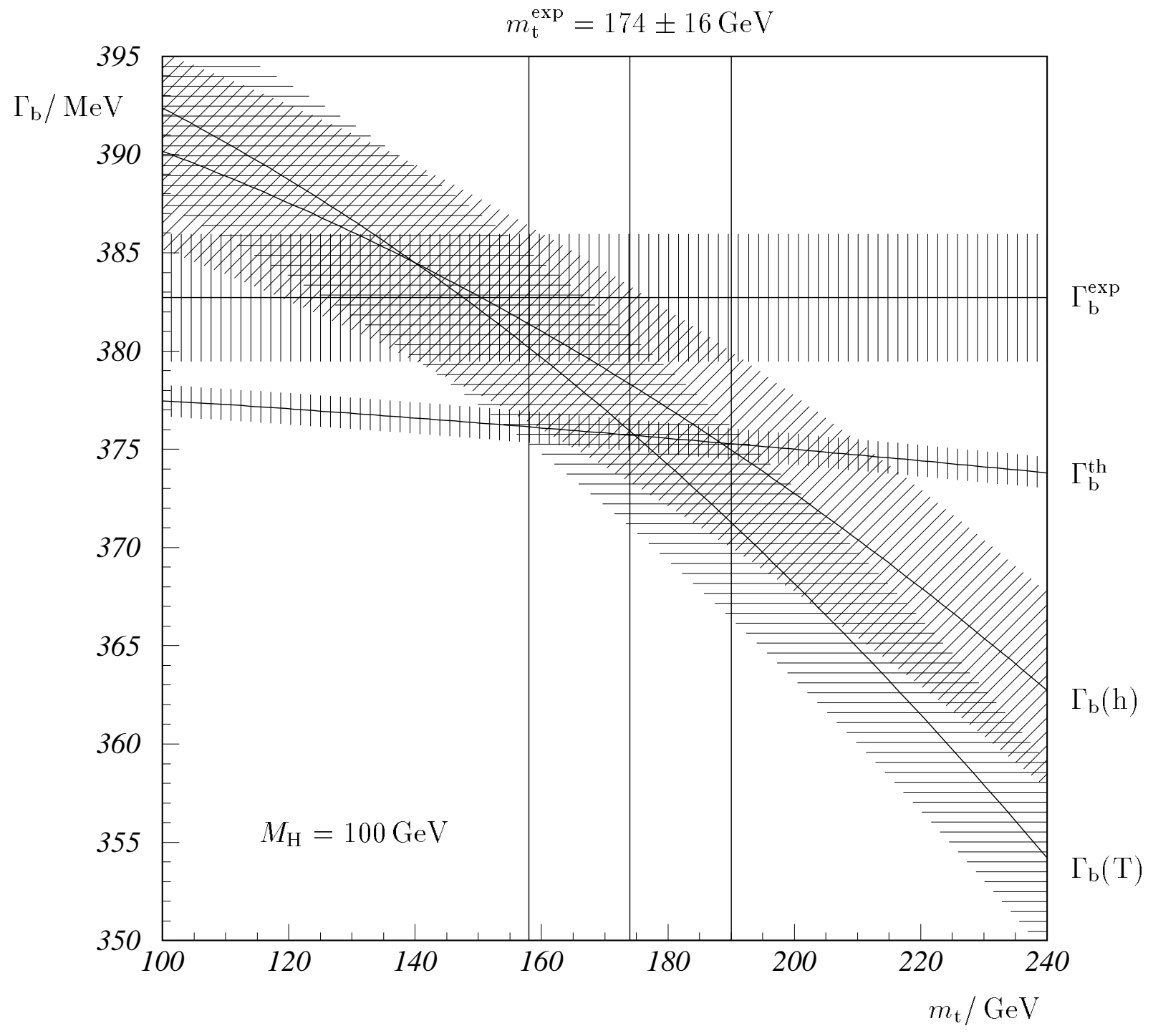

Figure 1: 


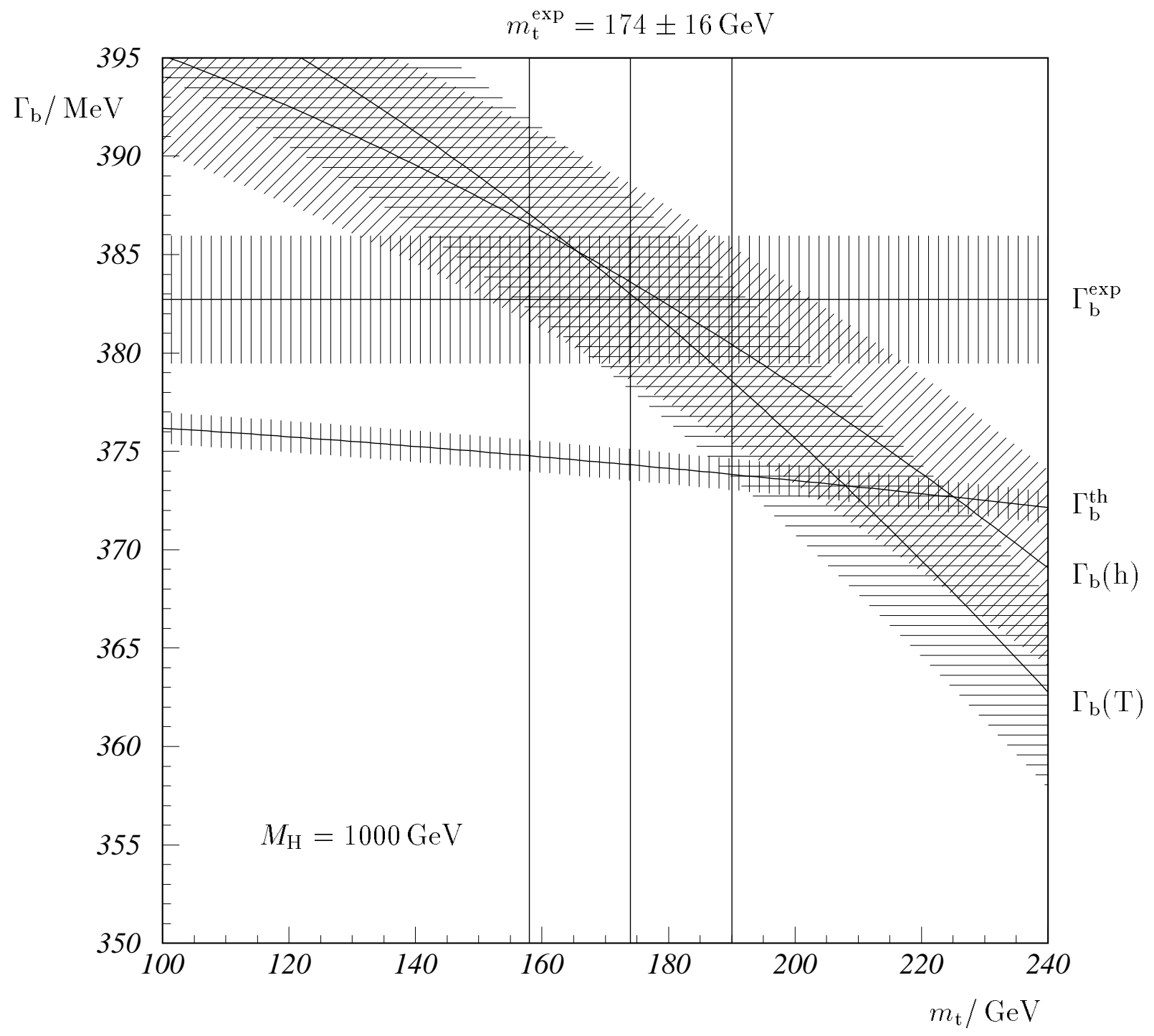

Figure 2: 


\title{
A Remark on the $Z^{0} \rightarrow b \bar{b}$ Width
}

\author{
D. Schildknecht \\ Department of Theoretical Physics \\ University of Bielefeld
}

\begin{abstract}
The $Z^{0} \rightarrow b \bar{b}$ width, $\Gamma_{b}$, is analysed in conjunction with the total and hadronic $Z^{0}$ widths, $\Gamma_{T}$ and $\Gamma_{h}$. Assuming, tentatively, that the present $2 \sigma$ discrepancy in $\Gamma_{b}$ will substantiate as time goes on, for large values of $m_{H}$ it will be sufficient to modify the $Z^{0} b \bar{b}$ vertex only. In contrast, for small values of $m_{H}$, the theoretical predictions for both the $Z^{0}$ width into light quarks and leptons as well as the $Z^{0} \rightarrow b \bar{b}$ vertex will have to be modified.
\end{abstract}


The precise agreement (e.g. ref. [1]) between the predictions of the $S U(2)_{L} \times U(1)_{Y}$ electroweak theory [2] and the experimental data [3] is remarkable indeed. The only evidence for a possible discrepancy between theory and experiment was found in the value of the $Z^{0} \rightarrow b \bar{b}$ width, which deviates from the theoretical prediction by approximately two standard deviations. The data are consistent with the width predicted for $Z^{0} \rightarrow d \bar{d}$, and accordingly, they do not show the effect expected from the presence of the mass of the heavy top quark in the $Z^{0} b \bar{b}$ vertex. As the discrepancy amounts to two standard deviations only, it may be wise to wait for further analysis of forthcoming data before reflecting too much on a possible theoretical explanation of it.

In the present note, nevertheless, we deal with the $Z^{0} \rightarrow b \bar{b}$ width, restricting ourselves, however, to a few general comments on how the $Z^{0} \rightarrow b \bar{b}$ "anomaly" could be accommodated in case it will substantiate and stand the test of time. We will biefly analyse the data on $\Gamma_{b}$ in conjunction with the data on the total and hadronic $Z^{0}$ widths, $\Gamma_{T}$ and $\Gamma_{h}$, respectively, in comparison with standard predictions. Our essential point consists of the observation that low and high values of the Higgs mass $m_{H}$, require different dominant modifications of the theory in order to accommodate the experimental value of $\Gamma_{b}$ in conjunction with the experimental data for $\Gamma_{T}$ and $\Gamma_{h}$.

Our analysis will be based on the experimental data presented at the Glasgow Conference $[3]$,

$$
\begin{aligned}
M_{Z} & =91.1888 \pm 0.0044 \mathrm{GeV}, \\
\Gamma_{T} & =2497.4 \pm 3.8 M e V, \\
R & =\Gamma_{h} / \Gamma_{l}=20.795 \pm 0.040, \\
\sigma_{h} & =\frac{12 \pi \Gamma_{l} \Gamma_{h}}{M_{Z}^{2} \Gamma_{T}^{2}}=41.49 \pm 0.12 n b .
\end{aligned}
$$

From the values of $R$ and $\sigma_{h}$ one derives [1] *

$$
\begin{gathered}
\Gamma_{l}=83.96 \pm 0.18 \mathrm{MeV}, \\
\Gamma_{h}=1746 \pm 4 \mathrm{MeV},
\end{gathered}
$$

\footnotetext{
* The correlation matrix between $\Gamma_{T}, R$ and $\sigma_{h}$ was taken into account.
} 
and from the measured value of $* *$

$$
R_{b h}=\frac{\Gamma_{b}}{\Gamma_{h}}=0.2192 \pm 0.0018
$$

one then obtains

$$
\Gamma_{b}=382.7 \pm 3.3 \mathrm{MeV}
$$

In what follows, we will compare the data for $\Gamma_{b}$ in conjunction with the ones for $\Gamma_{T}$ and $\Gamma_{h}$ with standard theoretical predictions. All three of these quantities can be simultaneously analysed in a unified manner by first of all extracting the $Z^{0} \rightarrow b \bar{b}$ width from the experimental total and hadronic widths, $\Gamma_{T}^{e x p}$ and $\Gamma_{h}^{e x p}$, respectively, via

$$
\Gamma_{b}(T) \equiv \Gamma_{T}^{e x p}-2\left(\Gamma_{u}^{t h}+\Gamma_{d}^{t h}\right)-3\left(\Gamma_{e}^{t h}+\Gamma_{\nu}^{t h}\right)
$$

and

$$
\Gamma_{b}(h) \equiv \Gamma_{h}^{e x p}-2\left(\Gamma_{u}^{t h}+\Gamma_{d}^{t h}\right)
$$

In these formulae, $\Gamma_{u}^{t h}, \Gamma_{d}^{t h}$, etc. denote the (radiatively corrected) theoretical partial $Z^{0}$ widths for the $Z^{0} \rightarrow u \bar{u}, Z^{0} \rightarrow d \bar{d}$, etc. decays, while $\Gamma_{b}(T)$ and $\Gamma_{b}(h)$ refer to the partial widths for the $Z^{0} \rightarrow b \bar{b}$ decay extracted from the total and hadronic $Z^{0}$ widths, $\Gamma_{T}$ and $\Gamma_{h}$, respectively. It is evident that $\Gamma_{b}(T)$ and $\Gamma_{b}(h)$ in $(5),(6)$, are "semi-experimental" quantities. They depend on the experimental data on the total and hadronic $Z^{0}$ widths, $\Gamma_{T}^{e x p}$ and $\Gamma_{h}^{e x p}$, as well as the theoretical predictions for the other partial $Z^{0}$ widths which are subtracted on the right-hand-sides in (5), (6). Due to the strong dependence on the mass of the top quark, $m_{t}$ (via the leading $m_{t}^{2}$ dependence), also $\Gamma_{b}(T)$ and $\Gamma_{b}(h)$ will be decreasing functions of $m_{t}$. In addition, $\Gamma_{b}(T)$ and $\Gamma_{b}(h)$ will depend on the Higgs mass, $m_{H}$, via $\ln m_{H}$.

Upon inserting the necessary theoretical partial widths into (5) and (6), we will compare $\Gamma_{b}(T)$ and $\Gamma_{b}(h)$ with the theoretical prediction for the $Z^{0} \rightarrow b \bar{b}$ width, $\Gamma_{b}^{t h}$, and with the experimental one, $\Gamma_{b}^{e x p}$, and draw our conclusions.

** This value of $R_{b h}$ is obtained [3] upon fixing $R_{c} \equiv \Gamma_{c} / \Gamma_{h}$ to its Standard Model value of $R_{c}=0.171$. 
The theoretical values for partial decay widths of the $Z^{0}$ into leptons and quarks are taken from our recent analysis of the electroweak precision data [1], based on

$$
\begin{aligned}
\alpha\left(M_{Z}^{2}\right)^{-1} & =128.87 \pm 0.12 \\
G_{\mu} & =1.16639(2) \cdot 10^{-5} \mathrm{GeV}
\end{aligned}
$$

as well as $M_{Z}$ from (1) and

$$
\begin{aligned}
& \alpha_{s}=0.118 \pm 0.007 \\
& m_{b}=4.5 \mathrm{GeV}
\end{aligned}
$$

as input parameters.

The results of the present analysis are presented in figs. 1,2 for the two cases of a low value of $m_{H}=100 \mathrm{GeV}$ and a high value of $m_{H}=1000 \mathrm{GeV}$, respectively.

We first of all consider the case of $m_{H}=100 \mathrm{GeV}$ shown in fig. 1 . From this figure one finds rough agreement of the $Z^{0} \rightarrow b \bar{b}$ width extracted from the total and hadronic widths with the theoretical prediction, $\Gamma_{b}^{t h}$, i.e.

$$
\Gamma_{b}(T) \cong \Gamma_{b}(h) \cong \Gamma_{b}^{t h}
$$

for

$$
\begin{gathered}
m_{t} \cong 175 \mathrm{GeV}, \\
m_{H} \cong 100 \mathrm{GeV} .
\end{gathered}
$$

Obviously, the result (9), (10) is nothing else but the (known) consistency between theory and experiment in the total $Z^{0}$ width and in the hadronic $Z^{0}$ width, expressed, however, in terms of the $Z^{0} \rightarrow b \bar{b}$ partial width. This consistency holds for values of $m_{t} \cong 175 \mathrm{GeV}$, the value favored by the results of the direct searches for the top quark [4.]. To remove the (indication of a small) discrepancy with $\Gamma_{b}^{e x p}$ in fig. 1, both, the theoretical prediction for $Z^{0} \rightarrow b \bar{b}$ decay, $\Gamma_{b}^{t h}$, as well as $\Gamma_{b}(T)$ and $\Gamma_{b}(h)$ will have to be modified, in order to keep the validity of (9). According to (5) and (6), this implies that the theoretical predictions for the $Z^{0}$ widths into light leptons and quarks will have to decrease. In summary, for small values of $m_{H}$, the data - always assuming that the minor discrepancy between theory and experiment visible at present will substantiate - require a modification of the theory which enlarges $\Gamma_{b}^{t h}$ and diminishes $\Gamma_{u}^{t h}, \Gamma_{d}^{t h}$, etc.

The situation (for $m_{t} \cong 175 \mathrm{GeV}$ ) is different in the case of the other extreme, a large mass of the Higgs boson of e.g. $m_{H}=1000 \mathrm{GeV}$, as shown in fig. 2. In contrast to (9) 
we now have

$$
\Gamma_{b}(T) \cong \Gamma_{b}(h) \cong \Gamma_{b}^{e x p}
$$

for

$$
\begin{gathered}
m_{t} \cong 175 \mathrm{GeV}, \\
m_{H} \cong 1000 \mathrm{GeV} .
\end{gathered}
$$

For large values of $m_{H}$ the (theoretical) values for the $Z^{0}$ widths into light quarks and leptons in (5), (6) are sufficiently suppressed to accommodate the present enhanced experimental value of $\Gamma_{b}^{e x p}$ within the total and hadronic widths, $\Gamma_{T}^{e x p}$ and $\Gamma_{h}^{e x p}$. Accordingly, in this case, it will be sufficient to modify the $Z^{0} b \bar{b}$ vertex to obtain consistency with the data for $\Gamma_{b}^{e x p}$ as well as $\Gamma_{T}^{e x p}$ and $\Gamma_{h}^{e x p}$.

In conclusion, the presentation of the data given in figs. 1, 2 clearly illustrates the delicate interplay of the different experimental results and the parameters $m_{t}$ and $m_{H}$. If the $2 \sigma$ effect in $\Gamma_{b}$ will stand the test of time, its theoretical explanation will have to discriminate between the low- $m_{H}$ and the high- $m_{H}$ options (always assuming $m_{t} \cong$ $175 \mathrm{GeV})$. For low values of $m_{H}$ the theoretical predictions for the $Z^{0}$ widths into the light quarks and leptons as well as the $Z^{0} \rightarrow b \bar{b}$ width will have to be modified. On the other hand, in the limit of large values of $m_{H}$, it will dominantly only be the theoretical prediction for the $Z^{0} \rightarrow b \bar{b}$ vertex which must be changed.

\section{Acknowledgement}

The author would like to thank Stefan Dittmaier for fruitful collaboration on electroweak interactions and help in the presentation of the results in figures 1, 2. 


\section{References}

[1] S. Dittmaier, D. Schildknecht, M. Kuroda, Bielefeld-preprint BI-TP 94/62, hep-ph/9501404.

[2] S.L. Glashow, Nucl.Phys.B 22 (1961) 579;

S. Weinberg, Phys.Rev.Lett. 19 (1967) 1264;

A. Salam, in: Elementary Particle Theory ed. N. Svartholm (Almquist and Wiksell, 1968), p. 367.

[3] D. Schaile, plenary talk given at the 27th International Conference of High Energy Physics, Glasgow, July 1994,

LEP collaborations, preprint CERN/PPE/94-187.

[4] F. Abe et al., CDF Collaboration, Phys.Rev. D50 (1995) 2966.

\section{Fig. 1:}

In addition to $\Gamma_{b}^{e x p}$, the figure shows $\Gamma_{b}^{t h}$ as a function of the mass of the top quark, $m_{t}$, as well as the "semi-experimental" quantities $\Gamma_{b}(T)$ and $\Gamma_{b}(h)$ obtained from the total and hadronic $Z^{0}$ widths, $\Gamma_{T}$ and $\Gamma_{h}$, by subtracting the theoretical predictions for the $Z^{0}$ decay widths into light quarks and leptons. The value of $m_{t}=174 \pm 16 \mathrm{GeV}$ preferred by the CDF searches is also indicated. For the theoretical prediction for $\Gamma_{b}^{t h}$ and for $\Gamma_{b}(T)$ and $\Gamma_{b}(h)$ a Higgs-boson of mass of $m_{H}=100 \mathrm{GeV}$ was adopted. The error in $\Gamma_{b}^{t h}$ is due to the experimental error in $\alpha_{s}$. This error is also taken into account in $\Gamma_{b}(T)$ and $\Gamma_{b}(h)$.

\section{Fig 2.:}

As fig 1, but for $m_{H}=1000 \mathrm{GeV}$. 